\title{
First-in-human topical microbiome transplantation with Roseomonas mucosa for atopic dermatitis
}

Ian A. Myles, ${ }^{1}$ Noah J. Earland, ${ }^{1}$ Erik D. Anderson, ${ }^{1}$ Ian N. Moore, ${ }^{2}$ Mark D. Kieh, ${ }^{1}$ Kelli W. Williams, ${ }^{1}$ Arhum Saleem, ${ }^{1}$ Natalia M. Fontecilla, ${ }^{1}$ Pamela A. Welch, ${ }^{1}$ Dirk A. Darnell, ${ }^{1}$ Lisa A. Barnhart, ${ }^{1}$ Ashleigh A. Sun, ${ }^{1}$ Gulbu Uzel, ${ }^{1}$ and Sandip K. Datta ${ }^{1}$

'Laboratory of Clinical Immunology and Microbiology, National Institute of Allergy and Infectious Diseases (NIAID), NIH, Bethesda, Maryland, USA. ${ }^{2}$ Infectious Disease and Pathogenesis Section, Comparative Medicine Branch, NIAID, NIH, Rockville, Maryland, USA.

The underlying pathology of atopic dermatitis (AD) includes impaired skin barrier function, susceptibility to Staphylococcus aureus skin infection, immune dysregulation, and cutaneous dysbiosis. Our recent investigation into the potential role of Gram-negative skin bacteria in AD revealed that isolates of one particular commensal, Roseomonas mucosa, collected from healthy volunteers (HVs) improved outcomes in mouse and cell culture models of AD. In contrast, isolates of $R$. mucosa from patients with AD worsened outcomes in these models. These preclinical results suggested that interventions targeting the microbiome could provide therapeutic benefit for patients with AD. As a first test of this hypothesis in humans, 10 adult and 5 pediatric patients were enrolled in an open-label phase I/II safety and activity trial (the Beginning Assessment of Cutaneous Treatment Efficacy for Roseomonas in Atopic Dermatitis trial; BACTERIAD I/II). Treatment with $R$. mucosa was associated with significant decreases in measures of disease severity, topical steroid requirement, and $S$. aureus burden. There were no adverse events or treatment complications. We additionally evaluated differentiating bacterial metabolites and topical exposures that may contribute to the skin dysbiosis associated with $A D$ and/or influence future microbiome-based treatments. These early results support continued evaluation of $R$. mucosa therapy with a placebo-controlled trial.

Authorship note: AS and NMF contributed equally to this work.

Conflict of interest: The authors have declared that no conflict of interest exists.

Submitted: March 21, 2018

Accepted: April 4, 2018

Published: May 3, 2018

Reference information: JCI Insight. 2018;3(9):e120608. https://doi.org/10.1172/jci. insight.120608.

\section{Introduction}

Atopic dermatitis (AD) is a highly prevalent inflammatory skin disease associated with reduced quality of life, increased health care expenditures, and an increased risk of developing asthma, allergic rhinitis, and food allergies $(1,2)$. The underlying pathology of $\mathrm{AD}$ includes impaired skin barrier function, susceptibility to Staphylococcus aureus skin infection, and immune dysregulation (3). Although AD is becoming increasingly more common in industrialized nations $(4,5)$, the natural history of $\mathrm{AD}$ has remained one in which the majority of patients will experience spontaneous remission before adolescence (6-8). Yet while the disease may be self-limited, the prolonged barrier defects predispose patients to cutaneous sensitization and the subsequent development of associated atopic diseases $(2,9)$. First-line therapies such as topical emollients, corticosteroids, PDE-4 inhibitors, and calcineurin inhibitors require multiple-times-per-day applications that negatively impact quality of life for patients and their families (10). The newly approved, biweekly biologic targeting IL-4Ra improves symptoms and lessens treatment demands, but its current $\$ 37,000$ annual price will make it inaccessible to many patients (11).

While contemporary studies have elucidated how $S$. aureus may directly exacerbate AD (12), both topical and prophylactic antibiotics have failed to show benefit and are not recommended by any consensus management guidelines $(1,13)$. We noted that in published microbiome studies the areas of skin characterized by carriage of Gram-negative bacteria (14) overlap with areas most commonly involved in AD (15), and this carriage is significantly decreased in AD patients compared with healthy controls (16). These findings suggest a role for the skin microbiota beyond $S$. aureus in the pathogenesis of AD. Our recent investigation into the potential role of Gram-negative skin bacteria in $\mathrm{AD}$ revealed that isolates of one particular 
commensal, Roseomonas mucosa, collected from healthy volunteers (HVs) improved outcomes in mouse and cell culture models of $\mathrm{AD}$ (17). In contrast, application of an $\mathrm{AD}$-sourced $R$. mucosa had either no impact on these modeled outcomes, or made the outcomes worse. These preclinical results generated the hypothesis that interventions targeting the microbiome could provide therapeutic benefit for patients with AD. As a first test of the therapeutic potential of topical live $R$. mucosa in humans, here we report the initial results from our clinical trial (NCT03018275) to preliminarily assess safety and activity. Treatment with $R$. mucosa was associated with a significant decrease in both objective disease measures and subjective pruritus without any reported adverse events or treatment complications. These results support the continued evaluation of this therapy with a placebo-controlled trial in patients with $\mathrm{AD}$.

\section{Results}

R. mucosa does not display signs of toxicity in mice. Case reports purporting $R$. mucosa infection are mostly limited to polymicrobial, catheter-associated infections in the setting of significant immune suppression (18). Notably, these reports never assessed whether the isolates of $R$. mucosa associated with clinical symptoms could fulfill Koch's postulates for defining pathogens. Our previous work found that $R$. muco$s a$ application to mouse skin that had undergone tape-stripping or chemical-induced dermatitis provided clinical benefit for the mice without any signs of toxicity (17). However, prior to clinical use we assessed the potential for systemic spread of $R$. mucosa to cause pathology by injecting mice intravenously with $10^{3}, 10^{4}$, or $10^{8}$ colony-forming units (CFU) of $3 \mathrm{HV}$-derived $R$. mucosa strains independently, a strain of R. mucosa (ATCC BAA-692) isolated from a clinical blood culture (19), or saline diluent alone. Animal technicians unaffiliated with the study monitored the mice in a blinded fashion for 10 days to assess weight loss, change in activity, moribundity, and mortality. There were no significant differences in mean weight change between groups exposed to diluent $(-6 \pm 75 \mathrm{mg}), 10^{3}(+3 \pm 63 \mathrm{mg}), 10^{4}(+4 \pm 93$ $\mathrm{mg})$, or $10^{8}(-2 \pm 83 \mathrm{mg}) \mathrm{CFU}$. There were no differences in activity or moribundity, and survival in all groups was $100 \%$. On day 10 after infection, the kidney, liver, and spleen were harvested for histologic examination. There were no differences in histologic appearance of the organs and no signs of infection or inflammatory changes were seen (Supplemental Figure 1; supplemental material available online with this article; https://doi.org/10.1172/jci.insight.120608DS1). Therefore, $R$. mucosa demonstrates low pathogenic potential.

$R$. mucosa was associated with clinical improvement and safety in adults. As a first-in-human test of the therapeutic potential of $R$. mucosa, 10 adults were enrolled in an open-label phase I/II trial (NCT03018275). Sucrose solutions containing escalating doses of live $R$. mucosa were topically applied twice weekly for 6 weeks, followed by a 4-week washout phase (Figure 1A). Patients were provided sufficient volumes for topical application to their bilateral antecubital fossae and one additional body surface area of their choice. AD severity was assessed using the SCORAD scale (20). Consistent with prior findings (16, 17), enrollment cultures of the patients' antecubital fossae did not yield Gram-negative bacteria.

All patients reported compliance with all 12 doses. Patients were given the phone number of the primary investigator to call with any unsolicited events. Solicited events were collected via a memory aide as well as remote visits via text, phone, or email. Solicited events included fever $\left(100.4^{\circ} \mathrm{F}\right.$ or greater), pain or tenderness (minimal even if not interfering with activity), and discoloration (slight and where treatment applied). No adverse reactions were reported (Supplemental Table 1).

Treatment was associated with significant reduction in objective intensity (Figure 2A), subjective regional pruritus (Figure $2 \mathrm{~B}$ and Supplemental Table 2), and antecubital-specific SCORAD (Figure 2C and Supplemental Table 2). Patients were instructed to maintain their home regimens (Supplemental Table 2) throughout active treatment. However, by the end of the washout phase, steroid-sparing effects of $R$. mucosa treatment were evident (Figure 2D). Some patients experienced responses at treated (but not untreated) sites outside of the antecubital region (Figure 2, E and F), suggesting that any passive transfer from treated to untreated sites was insufficient to generate a response. Responses were equal between the two sides when bilateral surfaces were treated (data not shown). Treatment of the hands was not associated with clinical benefit, even in patients with antecubital improvement (Figure 2F). Failure of $R$. mucosa to impact hand disease may indicate that higher treatment doses would be needed, a different species or strain of bacteria would be required, or that hand disease is not amenable to microbiome treatment. However, given the increased contact with topical antimicrobials on the hands, this finding may also be a consequence of environmental exposures promoting dysbiosis. 
A

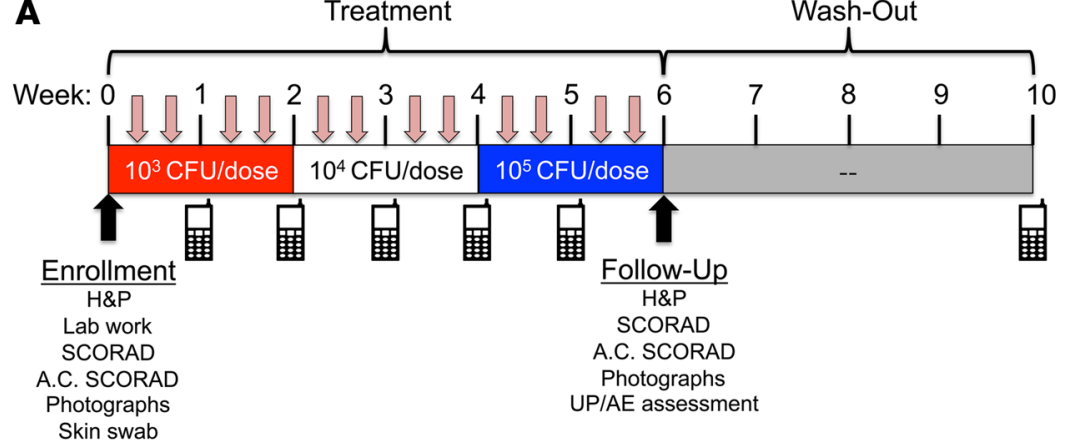

Figure 1. Overview of study design. Summary of study design for adult (A) and pediatric (B) cohorts. Upon enrollment patients underwent history and physical (H\&P), screening blood work, bacterial antecubital skin swab, and assessment of antecubital-specific (A.C.) and total SCORAD values. Patients self-administered the topical, live bacteria in a predetermined dose escalation from $10^{3}-10^{5}$ colony-forming units (CFU) per site. Treatments were twice per week (as indicated by pink arrows) for all adults and for weeks 0-12 for the pediatric cohort. Pediatric patients administered treatments every other day during weeks 13-16. Treatments consisted only of $R$. mucosa in $250 \mu \mathrm{l}$ of $10 \%-15 \%$ sucrose. Remote follow-up for solicitation of unexpected problems and/or adverse events (UP/AE; indicated by cell phone icons) was performed weekly during adult treatment and then 4 weeks after discontinuation of therapy. Washout evaluation for pediatric cohort is ongoing.

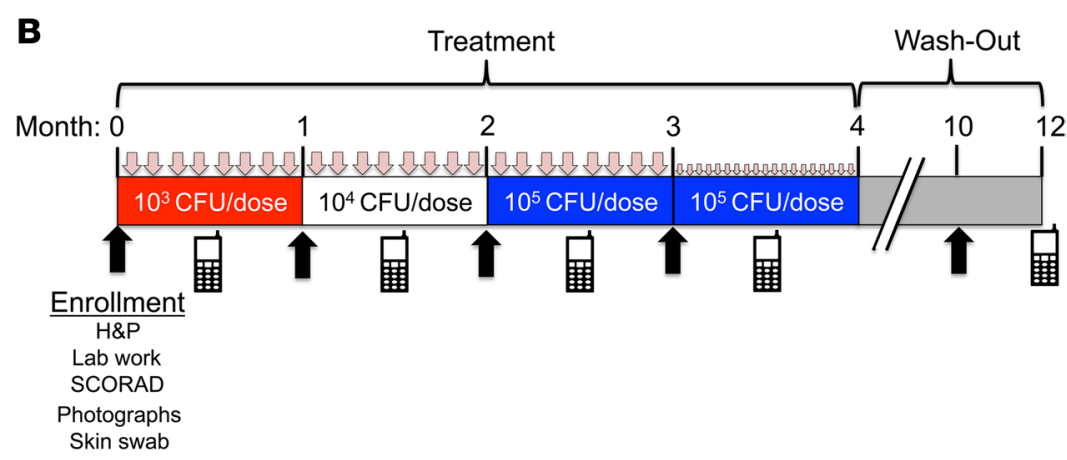

$R$. mucosa was associated with clinical improvement and safety in children. Based on the adult findings, we next enrolled 5 pediatric patients (aged 9-14 years) for a similar initial safety and activity evaluation (Figure 1B). Similar to the adult cohort, there were no solicited or unsolicited adverse events (Supplemental Table 1). In addition to solicited events for adult patients, pediatric patients were assessed for worsening of SCORAD ( $>20 \%$ increase in affected area from enrollment) and worsening of itching ( $>20 \%$ increase in pruritus score over enrollment). Laboratory values for complete blood count with differential, chemistry panel, hepatic enzymes, mineral panel, as well as erythrocyte sedimentation rate and C-reactive protein were tracked for the pediatric cohort and did not reveal any adverse changes (data not shown). The combined cohort was powered to detect an adverse event with a $10 \%$ true rate.

The pediatric cohort was treated twice weekly for 16 weeks and provided enough solution to treat all involved body surface areas. Parents reported full compliance with all treatment applications. Similar to the adult regional data, treatment of pediatric patients was associated with significant decreases in SCORAD (Figure 3, A and B), pruritus (Figure 3C), and steroid usage (Figure 3D). Furthermore, consistent with the adult data (Figure $2 \mathrm{~F}$ ) and reflected in the total SCORAD (Figure 3A), treatment outside of the antecubital region was also associated with improvement (Figure 3E). All pediatric patients were colonized with S. aureus (Figure 3F and Supplemental Figure 2, A and B). Consistent with our previous findings of direct anti-S. aureus effects of $R$. mucosa (17), treatment was associated with decreased $S$. aureus culture burden (Supplemental Figure 2, A and B) and the proportion of $S$. aureus relative to coagulase-negative staphylococci (CNS) in both the antecubital (Figure 3F) and popliteal fossae (Supplemental Figure 2C). The planned strain-level genomic analysis, as well as post-washout assessment, will better evaluate longterm treatment impacts on the microbiome as well as the colonization rate with the $R$. mucosa strains used in the therapeutic. Improvements in Children's and Family Dermatology Life Quality Index (CDLQI and FDLQI; Supplemental Figure 2, D and E), transepidermal water loss (TEWL; Supplemental Figure 2F), and IgE (Supplemental Table 2) did not meet statistical significance.

$R$. mucosa response associations were significantly greater than reported placebo effects. Noting a historical placebo effect of $5 \%-30 \%$, past studies have determined that a greater than $50 \%$ improvement in SCORAD (SCORAD-50) during treatment is statistically suggestive of treatment activity (21-24). By these parameters, treatment was associated with response in 4 pediatric patients (mean improvement of $-78.4 \%$ ) with one nonresponder $(-38 \%)$. Although regional SCORAD values for adults have not been specified previously, 
A
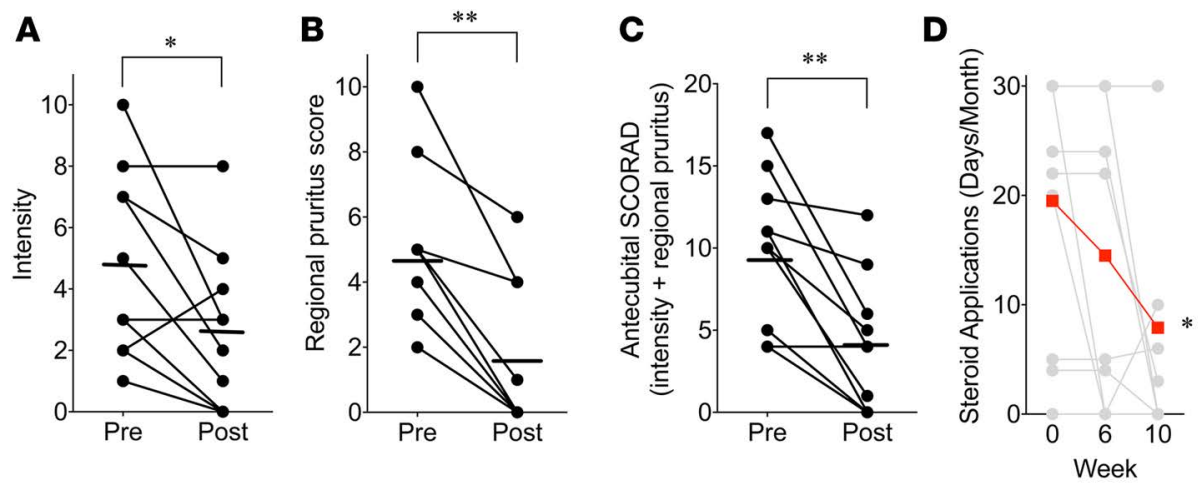

E
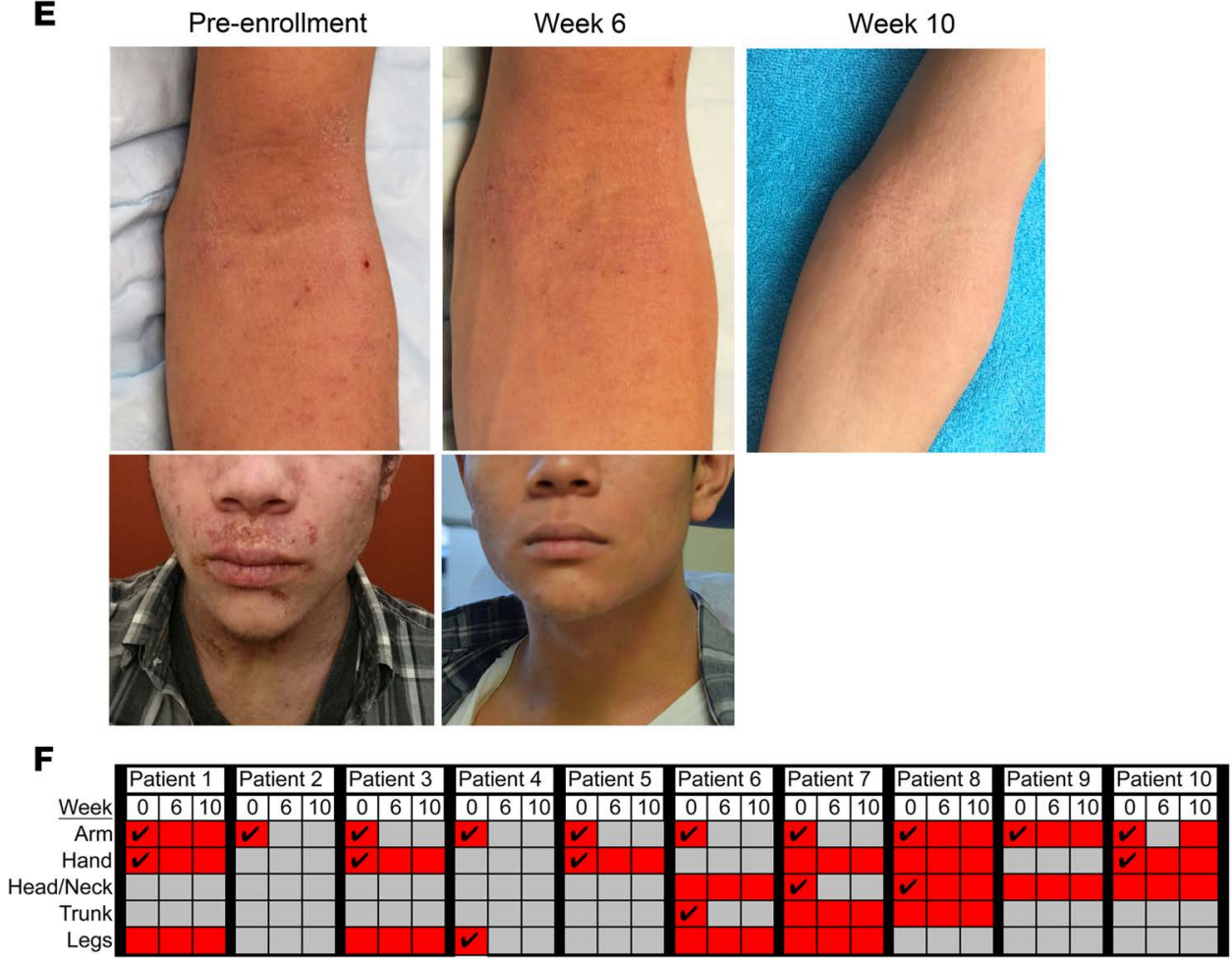

Figure 2. Topical Roseomonas mucosa shows activity against atopic dermatitis in adults. Mean (bars) and individual (circles; $n=10$ ) before- and after-treatment scores for objective intensity (A) and subjective pruritus (B) as measured by SCORAD. (C) Antecubital-specific SCORAD; sum of local intensity and pruritus scores. (D) Mean (scarlet) and individual (gray) self-reported steroid use (days/month) from the 6 weeks prior to enrollment (week 0$)$, after treatment (week 6), and after washout (week 10). Patients were instructed to maintain their home regimens throughout active treatment; however, patients 2 and 9 discontinued topical steroids upon initiation of $R$. mucosa treatment. (E) Pretreatment photos from enrollment, after treatment, and after washout (photo taken by patient) for the dominant-arm antecubital fossa and the face for patient 7 (see Supplemental Table 2). (F) Summary of areas of involvement (scarlet shading), asymptomatic areas (gray), and areas directly treated (check mark). Significance determined by 2-tailed Student's $t$ test and nonparametric Wilcoxon's matched-pairs test. ${ }^{*} P<0.05,{ }^{* *} P<0.01$.

extrapolating similar parameters designated 6 adult responders (-84.6\%), 1 partial responder $(-44 \%)$, and 3 nonresponders $(-8.6 \%)$. Further accounting for the natural history of $\operatorname{AD}(6,7,25)$, we calculated that fewer than 4 patients would achieve a SCORAD-50 during treatment (26.7\%) under the null hypothesis. Ten of the combined $15(66.7 \%)$ patients achieved the greater than $50 \%$ improvement threshold for regional or total SCORAD $(P=0.016)$. Furthermore, the rate of patients achieving $75 \%$ clinical improvement $(40 \%)$ significantly differed from reports of similar improvements attributable to placebo $(13.3 \% ; P=0.04)(23,24)$. The combined mean improvement for the cohort was $63.9 \%$; responders averaged $84.1 \%$ improvement and partial to nonresponders averaged $21.7 \%$ improvement. While differing in clinical scoring approaches, these results are consistent with previous open-label phase II studies on currently approved topical calcineurin and PDE-4 inhibitors $(26,27)$. In this trial, all adult patients who appeared responsive to treatment reported sustained or even additional clinical improvement after the washout phase (Figure 2, D and E). Continued enrollment and prolonged evaluation of pediatric participants after washout is ongoing. These data are suggestive of treatment activity and support continuation towards a placebo-controlled trial to confirm causation.

Lack of clinical response was associated with family history of skin disease. All patients designated as responders had a family history of atopy but did not report family histories of AD persisting into adulthood. However, of the 4 nonresponders, 3 (patient 1, 8, and P1) had family histories of AD persisting into adulthood in at least 3 generations (Supplemental Table 2). Patient 9 was raised by adoptive parents and did not know the medical history of her biologic relatives. The patient associated with partial response (patient 10) 
A

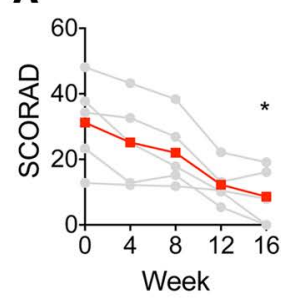

E

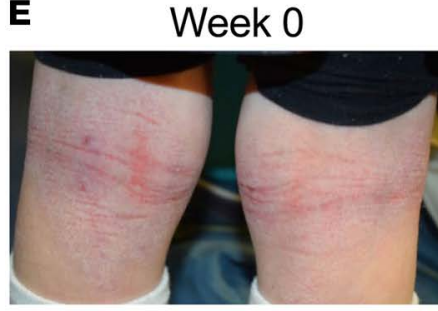

B

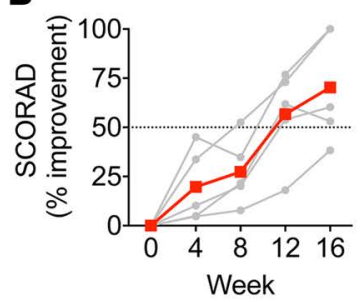

C

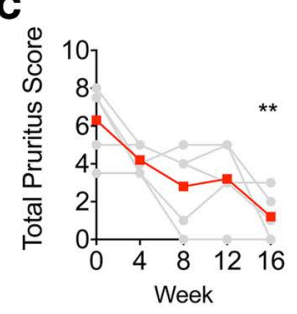

D

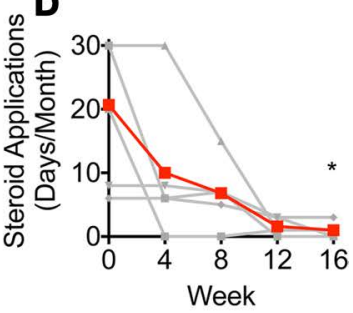

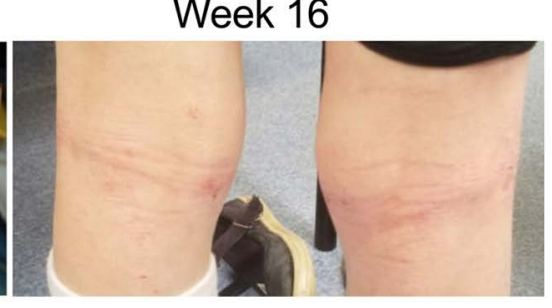

F

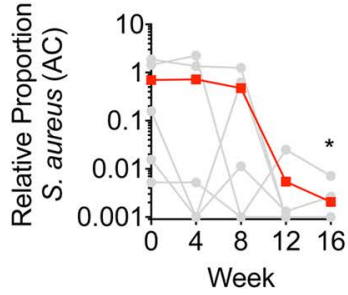

Figure 3. Topical Roseomonas mucosa shows activity against atopic dermatitis in children. Mean (scarlet) and individual (gray; $n=5$ ) SCORAD values (A) and percentage improvement (B) during treatment. Dotted line (B) indicates improvement level that is inconsistent with null hypothesis (see Methods). (C) Mean and individual pruritus. (D) Mean and individual patient-reported days of topical steroid use per month for the 3 months prior to enrollment (week 0 ) and during treatment. (E) Pretreatment photos from enrollment (week 0), after treatment (week 16) for the popliteal fossae for patient S5 (see Supplemental Table 1). (F) Ratio of Staphylococcus aureus to coagulase-negative staphylococci from the antecubital (AC) fossa as determined by culture. Significance determined by 2 -tailed Student's $t$ test and nonparametric Wilcoxon's matched-pairs test. ${ }^{*} P<0.05,{ }^{* *} P<0.01$ as determined versus enrollment value.

reported a history of hospital admission for $S$. aureus skin infection as an infant and a brother who died at age 3 due to $S$. aureus meningitis. Only one responder reported a family history of $\mathrm{AD}$ that persisted into adulthood, and no responders had a personal or family history of $S$. aureus infection. The association between these complex medical histories and the lack of clinical response suggests that differences in heritable host and/or microbial factors may impact treatment responses.

Metabolomic profiles differentiate strains of $R$. mucosa. Only 1 patient had culturable $R$. mucosa on enrollment (P2, Supplemental Table 2), consistent with prior genomic data of the staphylococcal predominance of active lesions (16). Our previous work in mouse and cell models established that $R$. mucosa isolates from HVs, but not from patients with $\mathrm{AD}$, improved key features of AD pathology (17). Under this hypothesis, even when $R$. mucosa is present on the unaffected skin of patients with $\mathrm{AD}$, strain-level differences may contribute to disease pathology and/or susceptibility to flares. To further evaluate if colonizing humans with specific strains of $R$. mucosa might offer targeted therapeutic utility, we assayed for differentiating metabolites that would be expected to improve established human pathways of AD pathogenesis (28). Our prior analysis on the bacterial supernatants demonstrated that $R$. mucosa strains differed in the production of lysophosphatidylcholine and cardiolipins (17). In the current analysis, reverse-phase liquid chromatography (RPLC) metabolomics analysis was performed on bacterial pellets (separated from the supernatants) of previously collected strains of $R$. mucosa from HVs versus strains from patients with AD (29) (Supplemental Figure 3, A and B). After correction for family-wise error rate (FWER), 6 metabolites distinguished strains from HVs from those from patients with $\mathrm{AD}$ (Supplemental Figure 3C). Strains from patients with $\mathrm{AD}$ produced the epithelial irritant mono-methyl glutarate (MMG) (30) as well as the histamine precursor histidinal. In contrast, strains from HVs contained phosphatidylcholine (PC 37:2) and phosphatidylethanolamine (PE 36:2, PE 14:0/20:1, and PE 22:1/14:1) (Supplemental Figure 3C). Similarly, hydrophilic interaction chromatography (HILIC) analysis demonstrated increased production of PC 38:2, PC 18:2/18:0, and PE-ceramide (d15:2[4E,6E]/20:0[2OH]) by HV-sourced $R$. mucosa isolates (Supplemental Figure 3D). However, statistical significance for HILIC-identified metabolites was lost after FWER adjustment. PC and $\mathrm{PE}$ are lipids known to enhance barrier function, provide direct and indirect protection against $S$. aureus, and modulate skin immunity $(17,31-34)$. Furthermore, while the bioactivities of PC and PE with these specific acyl chains have not been assessed, recent studies have demonstrated that abnormalities in skin lipid content and metabolism are strongly associated with $\mathrm{AD}(35,36)$. Taken together with our clinical findings, these data suggest that repairing the strain-level dysbiosis in $R$. mucosa in patients with AD could 
A

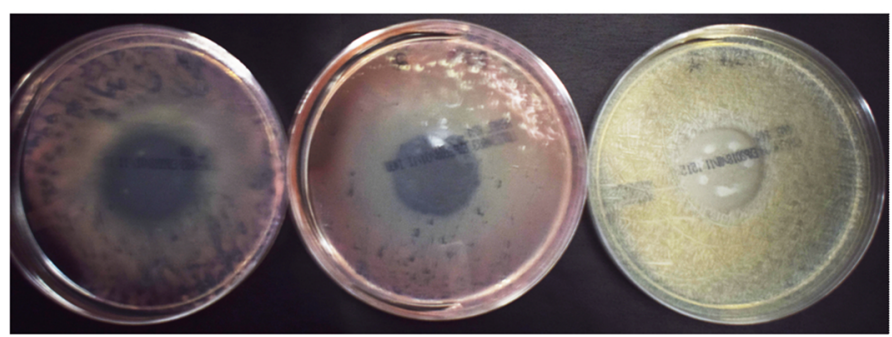

C

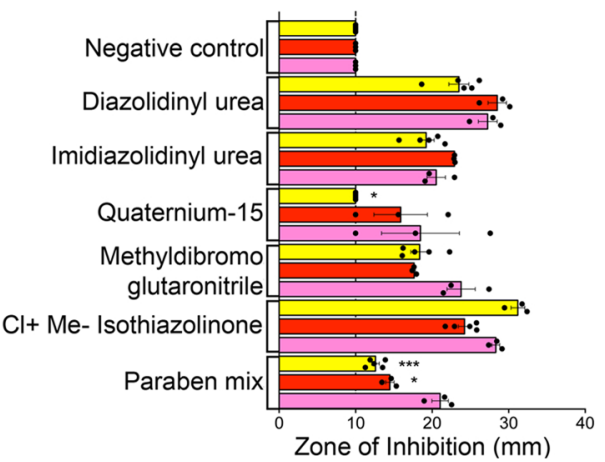

$\square$ S. aureus $\square R$. mucosa (AD) $\square R$. mucosa (HV)
D

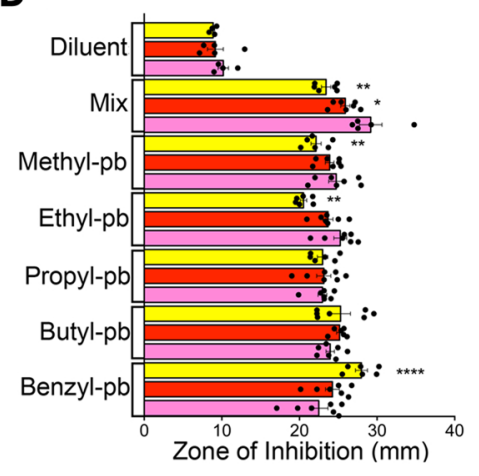

$\square$ S. aureus $\square R$. mucosa (AD) $\square R$. mucosa (HV)
B

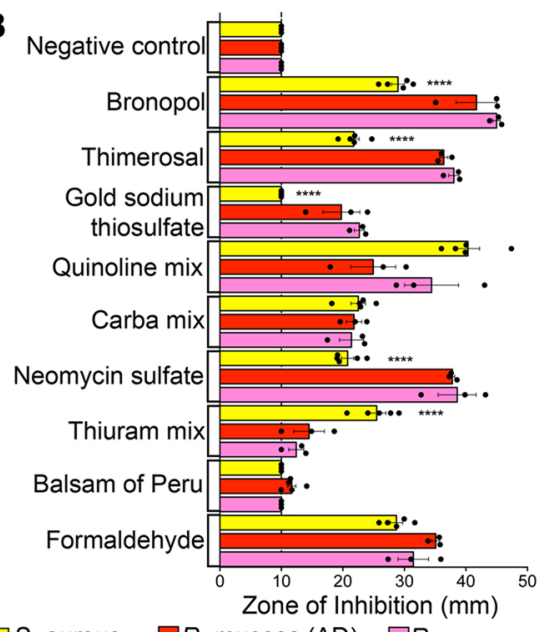

$\square$ S. aureus $\square R$. mucosa (AD) $\square R$. mucosa (HV)
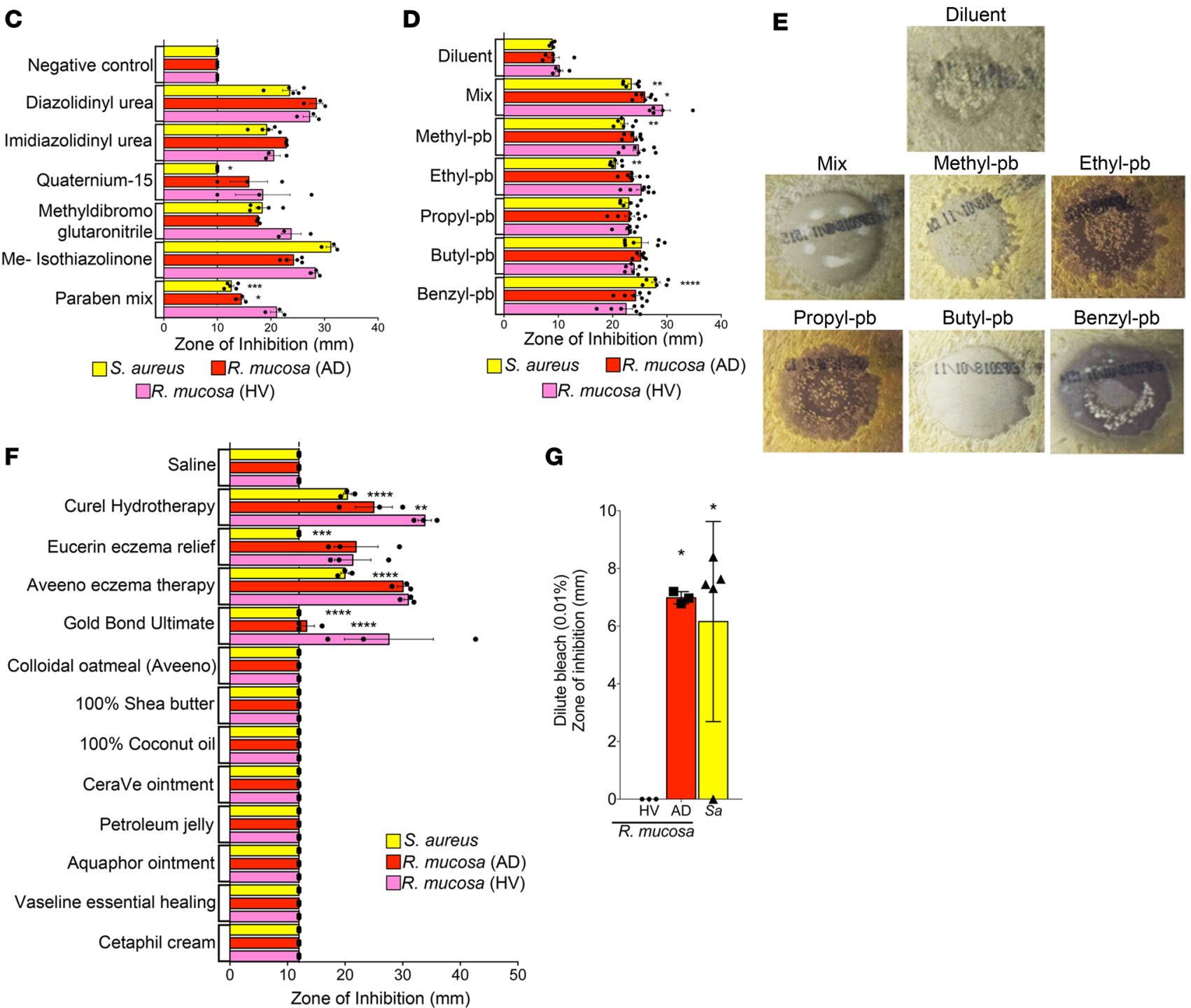

G

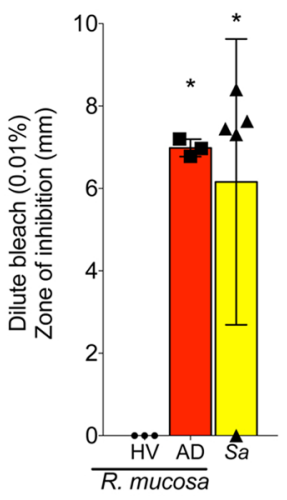

Figure 4. Strains of Roseomonas mucosa are impacted by environmental exposures. (A) Representative pictures for mean zone of inhibition (ZOI) on agar growth plates for R. mucosa from healthy volunteers (HVs), R. mucosa from patients with atopic dermatitis (AD), or Staphylococcus aureus. (B) Mean (bar) and individual (dots) ZOI for uncommon (B) and common (C) topical exposures. (D) Mean and individual ZOI for individual paraben (-pb) subtypes. (E) As in A, imaging of resistant S. aureus colonies in response to methyl-, ethyl-, propyl-, and benzyl-paraben exposure. (F) Mean and individual ZOI for 
common topical emollients. (G) Mean and individual ZOI for dilute bleach. Data are representative of 3 independent experiments using $n=3-7$ different isolates per group, per experiment and displayed as mean \pm SEM. ${ }^{*} P<0.05,{ }^{* *} P<0.01,{ }^{* * *} P<0.001,{ }^{* * * *} P<0.0001$ as determined by ANOVA, significance indicated as compared with $R$. mucosa (HV) group.

offer benefit to humans through enhanced production of barrier and immune-regulating phospholipids as well as reduction in the exposure to skin irritants.

$R$. mucosa growth is impacted by environmental exposures. While the open-label design typical for earlyphase clinical investigations precludes definitive statements on causation, our results as well as current literature suggest that the dysbiosis associated with AD may directly influence pathogenesis (37). To begin to elucidate environmental factors that may influence this dysbiosis, we used chemical-impregnated disks from a clinical patch test system to test the impacts of common topical exposures on bacterial growth (Figure 4A). Patch testing is designed to clinically evaluate contact dermatitis, and thus allows for testing the impacts of common topical exposures at concentrations reflective of real-world use. Many of the tested chemicals that inhibited $R$. mucosa growth are rare exposures (Figure 4B) that seem unlikely to contribute to a disease with a global lifetime risk of $5 \%-25 \%$ (38). However, some common preservatives inhibited the growth of $R$. mucosa more than $S$. aureus (Figure 4C). Paraben mix further inhibited HV strains of $R$. mucosa more than AD-associated $R$. mucosa and $S$. aureus (Figure $4 \mathrm{C}$ ). In response to individual parabens, susceptibility to inhibition (Figure 4D) and development of resistance (Figure 4E) differed between isolates. For $R$. mucosa, parabens also partially blanched the pink coloring (Figure 4A). Bacteria subcultured from the blanched sections remained viable and regained normal coloring after 48 hours (data not shown); therefore, the relevance of this discoloration remains to be elucidated. Additionally, while emollients are universally recommended for AD treatment (1), a select number of these products had significant differences in their growth inhibition of $R$. mucosa and $S$. aureus isolates (Figure $4 \mathrm{~F}$ ). In contrast, while the therapeutic guidelines and evidence of microbiome modification related to bleach baths differ $(1,39)$, dilute bleach inhibited $S$. aureus and disease-associated $R$. mucosa but not HV-sourced $R$. mucosa (Figure 4G).

\section{Discussion}

Current understanding of the microbiome's influence on human health has expanded far beyond the original hygiene hypothesis. Each new discovery identifies potential therapeutic approaches for improving treatment outcomes (40). These discoveries include successful phase II trials using a nontoxigenic strain of Clostridium difficile to prevent antibiotic-associated diarrhea and a natural isolate of Lactobacillus crispatus for urinary tract infection and bacterial vaginosis $(41,42)$. Emollients supplemented with lysates from the environmental Gram-negative Vitreoscilla filiformis demonstrate significantly greater benefit than placebo controls $(43,44)$. A more recent breakthrough revealed that strain-level differences in Staphylococcus epidermidis might impact the relative burden of $S$. aureus on the skin of patients with AD (37).

Our results are suggestive that strain-level differences in $R$. mucosa may directly influence AD and provide clinical benefit through multiple mechanisms that target epithelial barrier function, innate/adaptive immune balance, and $S$. aureus growth (17). Our metabolomics data further suggest lipid mediators and other biochemical correlates to target in future assessments of host skin metabolomic changes associated with therapy. Consistent with reported genomic risk factors in $\mathrm{AD}$ (45), the differing responses in patients with significant family histories suggest that heritable factors influence responses to $R$. mucosa therapy. However, while $R$. mucosa exposure was associated with clinical improvement, complete assessment of the microbiome before and after treatment in planned future trials will be required to more fully assess the role of $R$. mucosa in the pathogenesis of AD.

Future studies are also needed to better establish the conditions and exposures that promote and maintain the dysbiosis associated with $\mathrm{AD}$. Even if a given antimicrobial exposure inhibited $S$. aureus to an identical degree as commensal Gram-negative bacteria, due to staphylococci's significantly faster growth rate (29) such exposure would still provide $S$. aureus an advantage in repopulating the skin. However, both paraben mix and quatrium-15 inhibited $R$. mucosa to a significantly greater degree than $S$. aureus and select parabens differentially inhibited therapeutic $R$. mucosa isolates versus ADassociated strains. Yet while our data on topical products are provocative in relation to the increased rates of $\mathrm{AD}$ in the postindustrial era, many additional components of the exposome likely contribute to the cutaneous dysbiosis $(17,37,46)$. Furthermore, the relatively modern introduction along with 
the life-long ubiquity of these products disqualifies them from consideration as causative for a disease described in antiquity and most often limited to childhood (6-8).

A causal linkage for any of the tested compounds to $\mathrm{AD}$ symptoms, or dysbiosis itself, cannot be inferred from our findings. However, our environmental exposure data postulate that variations in topical products could exacerbate AD pathology by creating a microenvironment more favorable to growth of $S$. aureus and/or disease-associated strains of $R$. mucosa. Furthermore, such exposures may impact the effectiveness of potential microbiota-based therapeutic approaches. Informed recommendations on cutaneous exposures would benefit from creation of high-throughput systems to assay the impacts and synergy of topical products on both the survival and physiology of the microbiome.

Overall, our findings suggest the safety of topical $R$. mucosa therapy and justify continuation of our ongoing trial to assess safety and activity in a pediatric cohort of patients with $\mathrm{AD}$ (expanded to children aged 3-17 years). These studies will additionally assess changes in host serum markers, skin metabolomics, and the skin microbiota by culture and genomic methods. The results from our expanded pediatric cohort will help establish a causal and mechanistic basis for the promising preliminary benefit seen with topical microbiome transplantation, and lay foundations for larger, placebo-controlled trials that will be needed to definitively assess efficacy.

\section{Methods}

\section{Patients}

Patients were recruited and enrolled at the NIH under clinical trial NCT03018275 (Beginning Assessment of Cutaneous Treatment Efficacy of Roseomonas in Atopic Dermatitis, Phase I/II; BACTERiAD I/II). Prior to enrollment all patients and/or legal guardians signed informed consent. Pediatric patients signed assents. SCORAD (an established scoring algorithm for atopic dermatitis) values were determined under standard approaches (20). Two investigators documented the surface areas involved and intensity of disease. One investigator was intentionally misinformed that the study was a placebo-controlled design and therefore was unaware that all patients were on active treatment with the investigational drug. Only scores from the blinded investigator are shown. Patients provided the subjective values for pruritus and sleep disturbance. To meet inclusion criteria, patients needed to have a SCORAD value of 10 or higher, have disease present on the antecubital fossae and/or forearms, and have previously attempted standard of care therapy. Values for the antecubital specific SCORAD were obtained by adding the intensity values for the antecubital region (score 0-3 for dryness, erythema, edema, oozing, excoriation, and lichenification) to the patient-reported subjective score (score 0-10) for pruritus of the antecubital region. Antecubital fossae were swabbed for the presence of Gram-negative bacteria as previously described (17, 29). S. aureus and CNS burden was determined by vortexing swabs in $2 \mathrm{ml}$ of typsin broth (Remel) for 30 seconds and plating $100 \mu 1$ on blood agar plates (Remel). The following day, the number of colonies was enumerated and multiplied by 20 to obtain the total CFU in the 2-ml collection volume and then averaging values between both arms. Relative abundance of $S$. aureus was obtained by dividing the colony numbers for $S$. aureus by those for CNS.

Screening blood work included complete blood count, chemistry panel, calcium, magnesium, phosphorus, alkaline phosphatase, aspartate aminotransferase, alanine aminotransferase, bilirubin, albumin, and HIV antibodies for adults. Urine pregnancy testing was performed on all adult female patients. There were no restrictions on home medication use except for those specified in the exclusion criteria. Adult patients were instructed to make no changes in their home regimen. Pediatric patients were asked to maintain their standard approach; parents reported using daily emollients but reserving steroid treatments for flares. Parents were asked to recall average days/month of topical steroid use for the 3 months prior to treatment and then prospectively track steroid use during treatment. Transepidermal water loss was measured by VapoMeter (Delfin) per the manufacturer's instructions and represents the average results of the bilateral antecubital fossae.

\section{Inclusion criteria}

Inclusion criteria were (a) age 18 years or older (adult cohort) or age 7-17 (pediatric cohort); (b) SCORAD of at least 10; (c) carry a physician diagnosis of $\mathrm{AD}$ with active involvement of the antecubital fossa; (d) willing to allow storage of blood for future research; (e) no history of other skin disease; (f) initiated 
or attempted standard of care therapy at least 6 months prior to enrollment; and (g) agreement to use adequate contraception if indicated.

\section{Exclusion criteria}

Exclusion criteria were (a) presence of an indwelling venous or arterial catheter; (b) individuals living with anyone with a diagnosed immunodeficiency, cardiac valvular disease, and/or indwelling catheter; (c) presence of allergies to amikacin, ciprofloxacin, gentamicin, levofloxacin, and tobramycin (which would preclude treatment of any unexpected infection); (d) history of cardiac valvular disease; (e) any history of grade 2 or higher neutropenia or leukopenia; (f) clinical suspicion of immunodeficiency, liver disorder, kidney disorder, and/or HIV; (g) pregnant or breastfeeding; (h) any history of anti-tumor necrosis factor (TNF) treatment; (i) inability to demonstrate proper bacteria administration procedure despite coaching and training; (j) use of any antibiotics within 4 weeks of enrollment; (k) use of oral steroids within 4 weeks of enrollment; and (1) any condition that, in the opinion of the investigator, contraindicates participation in this study.

\section{Endpoints}

Primary endpoints were (a) frequency of solicited and unsolicited adverse events, serious adverse events, and death; and (b) 50\% reduction in regional or total SCORAD.

Secondary endpoints (applicable only to the pediatric cohort) were (a) 30\% improvement in the Children's Dermatology Life Quality Index (CDLQI); and (b) 30\% improvement in the Family Dermatology Life Quality Index (FDLQI).

\section{Qualitative analysis}

Patient statements at intake and follow-up visits were documented and examined for thematic consistencies. Commentary on body sites treated, duration of benefit, ease of use, doses missed, overall satisfaction, and adverse reactions were compiled and analyzed.

\section{Sample size derivations}

Safety. Sample size calculations demonstrated that with 15 total patients in the dose escalation portion ( 10 adults, 5 children), there would be a probability of 0.14 of observing 1 or more serious adverse events (SAEs) or AEs (grade 2 or higher as defined by the DAIDS toxicity table) if the true rate were 0.01 , and a probability of 0.8 of observing 1 or higher if the true rate were 0.1 by binomial distribution. An independent safety monitor assessed all clinical and laboratory data.

Activity. A 50\% reduction in SCORAD or regional SCORAD (SCORAD-50) was used as per prior publications $(21,24,47,48)$. The placebo response in SCORAD was calculated from placebo control data as approximately 5\%-30\% over studies lasting 1-12 months $(22,24,49-51)$. The natural history of AD suggests that $50 \%-90 \%$ of patents will self-resolve by approximately $12-15$ years of age $(6,7,25)$. The pace at which a cohort more than 7 years of age would be expected to outgrow their disease is less than $5 \%$ per year based on previous reports $(6,7,25)$. Thus, we calculated no more than 2 of our cohort to self-resolve over the course of a year; $<2 \%$ of a cohort would be expected to self-resolve during the 6 - to 16 -week treatment timeframe. With 15 total participants, if 4 successes were observed at the end of the study then there would be a 0.9 probability ( $90 \%$ power) of concluding the treatment were active if the true activity rate were 0.4 . These calculations were based on the binomial distribution.

\section{R. mucosa pharmaceutical formulation}

Preparations of $R$. mucosa for clinical use were produced under investigational new drug (IND) application 17303 from the Food and Drug Administration (FDA). Three isolates of $R$. mucosa taken from 3 HVs were grown in minimal media (R2A broth, Teknova; or Hanks Buffered Salt Solution, HBSS, Gibco) for 24-48 hours. Isolates were selected based on their ability to inhibit the growth of S. aureus, activate vitamin D pathways in human keratinocytes, and improve outcomes in mouse models of AD (17). Genomic sequencing was performed on all strains to verify that no transmittable, clinically significant antibiotic resistance genes were present. SNP-level assessment of strains is ongoing. The bacterial cells were washed 3 times in PBS (Gibco) and resuspended into 10\%-15\% sucrose in water for a concentration of $10^{9} \mathrm{CFU} /$ $\mathrm{ml}$ based on previously reported growth parameters (29). Serial dilutions were performed in $10 \%-15 \%$ 
sucrose to generate stocks of $10^{4}, 10^{5}$, and $10^{6}$ per ml. Aliquots of diluted bacterial samples were plated on R2A agar (Remel) and incubated at $32^{\circ} \mathrm{C}$ for $48-72$ hours to enumerate prelyophilization CFU concentration. Starting CFU values were $90 \%-105 \%$ of expected concentrations. Eight hundred microliters (adult) or $1.5 \mathrm{ml}$ (pediatric) of bacterial solution was frozen in $1.5-\mathrm{ml}$ amber glass vials (Wheaton; adult) or a 3-ml self-contained sprayer system (Discount Vials; pediatrics) prior to lyophilization (Labconco). Vials/ sprayers were sealed, labeled, and stored at $-70^{\circ} \mathrm{C}$ until dispensed to the patients. Three aliquots per batch were reconstituted in sterile water and plated after serial dilution to enumerate postlyophilization CFU concentration. Survival was $93 \%-99 \%$ of starting CFU after lyophilization. These aliquots were also plated on soybean-casein digest agar (BD Bioscience), Sabouraud dextrose agar (Remel), MacConkey agar (BD Bioscience), xylose lysine agar (Remel), charcoal agar (BD Bioscience), and mannitol salt agar (Remel) and assessed for the presence of contaminating bacteria as per USP 61/62. No contamination was found in any batches of Roseomonas treatment. To avoid batch effects, all doses were derived and vialed in one session.

\section{R. mucosa dosing and application}

Initial dosing was based on the lowest dose with activity in prior mouse models (17). Twice weekly doses were selected due to the slow growth to $R$. mucosa and a desire to avoid applying new bacteria before the prior dose would reach stationary growth phase (29). Adult patients received vials of $2 \mathrm{ml}$ of sterile water (Wheaton). Adults were trained on aspirating $800 \mu \mathrm{l}$ of water using a 1-ml syringe (BD Bioscience). The $800 \mu 1$ of water was injected into vials of the lyophilized bacteria, the stopper was replaced, and the vials were gently shaken for approximately 1 minute. The patients then aspirated 200-250 $\mu 1$ of the reconstituted bacterial solution and placed an atomizer tip on the syringe (MAD300, Teleflex). The solution was sprayed on the antecubital/forearm region of one arm, then repeated on the opposing side. The patients were allowed to use the remaining $300-400 \mu 1$ of solution on additional body surface areas so long as they documented which areas they treated. In total, patients administered 1 application, twice per week, for a total of 6 weeks. During the initial 2 weeks, patients reconstituted vials of $10^{4} \mathrm{CFU} / \mathrm{ml}$; therefore, application of $200-250 \mu \mathrm{l}$ indicated a treatment dose of $2 \times 10^{3}$ to $2.5 \times 10^{3}$ total bacterial colonies per surface area. After remote follow-up was performed to assure no complications with treatment, the patients progressed to 2 weeks of treatment with $2 \times 10^{4}$ to $2.5 \times 10^{4}$ bacterial colonies per surface area, then finally 2 $\times 10^{5}$ to $2.5 \times 10^{5}$ total bacterial colonies per surface area. Patients returned for clinical assessment after completion of the full 6 weeks of therapy and were contacted remotely 30 days after treatment. Patients received hands-on training prior to dispensing the medication and were provided with an instructional video for later reference (https://youtu.be/LkWavevg22w).

Pediatric patients were provided bacteria lyophilized in a self-contained sprayer system (Discount Vials). Eyedroppers (United States Plastic Corp) of $1.5 \mathrm{ml}$ sterile water were provided. For each dose, patients or their parents were instructed to empty the contents of the eyedropper into the sprayer vial, wait 2-5 minutes for reconstitution, and then spray. Sprayers were metered so that 3 pump sprays mirrored the $250 \mu \mathrm{l}$ applied in the adult trial. Dose concentrations of CFU/ml were identical to adult dosing. Similar to adults pediatric patients applied the treatment twice weekly for the first 3 months of treatment, then every other day for the final month. Dose escalations were every 4 weeks after safety assessments.

\section{Bacterial growth assessment}

For patch assay evaluation, $10^{9} \mathrm{CFU}$ of $R$. mucosa or $S$. aureus were plated on R2A agar (Remel). Individual TRUETest (SmartPractice) plastic challenge squares were removed from the adhesive backing with sterile forceps under sterile conditions. The squares were placed in the center of a plate immediately after plating of bacteria. Plates for $R$. mucosa were incubated at $32^{\circ} \mathrm{C}$ for 48 hours before measuring resultant zone of inhibition with the electronic caliper (Mitutoyo America); plates of $S$. aureus were incubated at $32^{\circ} \mathrm{C}$ overnight prior to measurement. For studies evaluating parabens individually, methyl-, ethyl-, propyl, butyl-, and benzyl-4-hydroxybutyrate (Sigma-Aldrich) were suspended in 100\% ethanol. To mirror the $1 \mathrm{mg} /$ patch of the standardized disk, each individual paraben was diluted to a final concentration of $200 \mu \mathrm{g} / 10 \mu \mathrm{l}$. With a sterile pipette, $10 \mu 1$ of each mix was placed in the center of an R2A agar plate coated with $10^{9} \mathrm{CFU}$ of $R$. mucosa or $S$. aureus. Blanched as well as potentially paraben-resistant isolates were subcultured by taking a sterile loop and plating on R2A agar — viability was assessed by presence of growth by 48 hours. Studies using commercial topical emollients were performed by placing $70 \mu \mathrm{g}$ of each product onto a sterile circular coverslip and then placing the cov- 
erslip onto the agar culture as above. Colloidal oatmeal was diluted to $300 \mathrm{mg} / \mathrm{ml}, 20 \mu 1$ was placed in the center of the agar plate and covered with a 12-mm cover slip as above. Pictures were taken with a Nikon D5300; contrast and brightness were enhanced evenly across all plates to aide visualization.

\section{Bacterial metabolomics}

The 3 strains of $R$. mucosa used in the clinical treatment formulation as well as 3 strains from patients with $\mathrm{AD}$ were isolated and enumerated as previously described $(17,29)$. Frozen pellets of $10^{9} \mathrm{CFU}$ were sent to The Proteomics \& Mass Spectrometry Facility at the Danforth Plant Science Center as arranged through Science Exchange. Samples were analyzed using LC-MS by HILIC chromatography on a 0.5 $\times 150 \mathrm{~mm}$ Zic-pHILIC column using $10 \mathrm{mM} \mathrm{NH}_{4} \mathrm{HCO}_{3}$ in water (A) and $10 \mathrm{mM} \mathrm{NH}_{4} \mathrm{HCO}_{3}$ in $95 \%$ acetonitrile (B) as solvents and by RPLC chromatography using the same solvents and a $0.5 \times 100 \mathrm{~mm}$ PLRPS column. The samples were extracted with $200 \mu 1$ of $80 \%$ methanol by vortexing for 10 minutes. The solids were collected by centrifugation, and then the supernatant was filtered through a $0.8-\mu \mathrm{m}$ spin filter. Samples were injected onto the LC-MS and data were acquired in polarity switching mode with data-dependent acquisition of MS/MS spectra. Data analysis was performed on Elements for metabolomics (Proteome Software Inc.). The ID Score was set to 0.8 , the $\log _{10}$ Intensity threshold was set to 7, the Minimum Number of Samples, was set to 3. After the thresholds were established, the statistical differences between the healthy and disease samples were tested with an analysis of variance (ANOVA) among the precursor intensities of the identified metabolites. The ANOVA used a standard BenjaminiHochberg procedure to control for the false discovery rate (FDR), at a significance level of $q=0.05$. Then, a FWER correction was applied.

Mice

Experiments were performed in both male and female mice, but age and sex matched within each experiment. C57BL/ 6 mice were purchased from Jackson Laboratories. Intravenous injection with saline diluent or $R$. mucosa was performed when mice were 7-8 weeks of age. Weights were taken on days $0,3,6$, and 10. On day 10 , mice were sacrificed and the right kidney, spleen, and liver were removed. Tissues were processed, sectioned, and stained with hematoxylin and eosin (H\&E) (HistoServ, Inc.). All tissues were evaluated by a board-certified veterinary pathologist and photomicrographs were taken using an Olympus BX51 microscope and Olympus DP73 camera.

\section{Statistics}

Significance was calculated by 2-tailed paired Student's $t$ test with Prism software (GraphPad). Calculations were repeated using nonparametric assumptions under Wilcoxon's matched-pairs test assumptions. The larger $P$ value from these calculations is presented. A $P$ value of less than 0.05 was considered significant.

\section{Study approval}

Studies in humans were conducted under registered clinical trial NCT03018275 after approval from the NIAID institutional review board. All subjects were provided informed consent prior to their participation in the study. All murine experiments were done in compliance with the guidelines of the NIAID Institutional Animal Care and Use Committee.

\section{Author contributions}

IAM designed the experiments, evaluated patients, and wrote the manuscript. NJE and EDA assisted on select experiments and wrote the manuscript. INM performed all work related to histologic evaluation of mice. MDK assisted on select experiments. KWW assisted with patient evaluation and sample collection. AS and NMF assisted with background research. PAW, DAD, and LAB provided administrative assistance. AAS provided administrative assistance and performed blinded assessment of patients. GU provided oversight of the project. SKD supervised the project and wrote the manuscript.

\section{Acknowledgments}

We would like to thank the patients and their families for their assistance in this project. We would like to thank Benjamin Snow and Kristin Young for administrative assistance, Jenna Bergerson for clinical oversight, and Sally Hunsberger for statistical guidance. We would also like to thank the NIAID building 33 
veterinarians, animal care, and breeder technicians for their assistance as well as Mr. and Mrs. Topolino (NIAID) for their sacrifice during the course of this project. This work was supported by the Intramural Research Program of the NIAID and the NIH.

Address correspondence to: Ian A. Myles, 9000 Rockville Pike, Building 10, Room 11S253, Bethesda, Maryland 20892, USA. Phone: 301.451.8420; Email: mylesi@niaid.nih.gov.

1. Eichenfield LF, Ahluwalia J, Waldman A, Borok J, Udkoff J, Boguniewicz M. Current guidelines for the evaluation and management of atopic dermatitis: A comparison of the Joint Task Force Practice Parameter and American Academy of Dermatology guidelines. J Allergy Clin Immunol. 2017;139(4S):S49-S57.

2. Bantz SK, Zhu Z, Zheng T. The atopic march: progression from atopic dermatitis to allergic rhinitis and asthma. J Clin Cell Immunol. 2014;5:202.

3. Boguniewicz M, Leung DY. Recent insights into atopic dermatitis and implications for management of infectious complications. J Allergy Clin Immunol. 2010;125(1):4-13.

4. Latvala J, von Hertzen L, Lindholm H, Haahtela T. Trends in prevalence of asthma and allergy in Finnish young men: nationwide study, 1966-2003. BMJ. 2005;330(7501):1186-1187.

5. Silverberg JI. Public health burden and epidemiology of atopic dermatitis. Dermatol Clin. 2017;35(3):283-289.

6. Margolis JS, Abuabara K, Bilker W, Hoffstad O, Margolis DJ. Persistence of mild to moderate atopic dermatitis. JAMA Dermatol. 2014;150(6):593-600.

7. Williams HC, Strachan DP. The natural history of childhood eczema: observations from the British 1958 birth cohort study. $\mathrm{Br}$ J Dermatol. 1998;139(5):834-839.

8 . Henderson J, et al. The burden of disease associated with filaggrin mutations: a population-based, longitudinal birth cohort study. J Allergy Clin Immunol. 2008;121(4):872-7.e9.

9. Pyun BY. Natural history and risk factors of atopic dermatitis in children. Allergy Asthma Immunol Res. 2015;7(2):101-105.

10. Lewis-Jones S. Quality of life and childhood atopic dermatitis: the misery of living with childhood eczema. Int J Clin Pract. 2006;60(8):984-992.

11. Dolgin E. First eczema biologic debuts but price could restrict use. Nat Biotechnol. 2017;35(5):391-392.

12. Park KD, Pak SC, Park KK. The pathogenetic effect of natural and bacterial toxins on atopic dermatitis. Toxins (Basel) 2016;9(1):3.

13. Eichenfield LF, et al. Guidelines of care for the management of atopic dermatitis: section 2. Management and treatment of atopic dermatitis with topical therapies. J Am Acad Dermatol. 2014;71(1):116-132.

14. Grice EA, Segre JA. The skin microbiome. Nat Rev Microbiol. 2011;9(4):244-253.

15. Lyons JJ, Milner JD, Stone KD. Atopic dermatitis in children: clinical features, pathophysiology, and treatment. Immunol Allergy Clin North Am. 2015;35(1):161-183.

16. Kong HH, et al. Temporal shifts in the skin microbiome associated with disease flares and treatment in children with atopic dermatitis. Genome Res. 2012;22(5):850-859.

17. Myles IA, et al. Transplantation of human skin microbiota in models of atopic dermatitis. JCI Insight. 2016;1(10):e86955.

18. Dé I, Rolston KV, Han XY. Clinical significance of Roseomonas species isolated from catheter and blood samples: analysis of 36 cases in patients with cancer. Clin Infect Dis. 2004;38(11):1579-1584.

19. Han XY, Pham AS, Tarrand JJ, Rolston KV, Helsel LO, Levett PN. Bacteriologic characterization of 36 strains of Roseomonas species and proposal of Roseomonas mucosa sp nov and Roseomonas gilardii subsp rosea subsp nov. Am J Clin Pathol. 2003;120(2):256-264.

20. Kunz B, Oranje AP, Labrèze L, Stalder JF, Ring J, Taïeb A. Clinical validation and guidelines for the SCORAD index: consensus report of the European Task Force on Atopic Dermatitis. Dermatology (Basel). 1997;195(1):10-19.

21. Leloup P, Stalder JF, Barbarot S. Outpatient home-based wet wrap dressings with topical steroids with children with severe recalcitrant atopic dermatitis: a feasibility pilot study. Pediatr Dermatol. 2015;32(4):e177-e178.

22. Rosenfeldt V, et al. Effect of probiotic Lactobacillus strains in children with atopic dermatitis. J Allergy Clin Immunol. 2003;111(2):389-395.

23. Simpson EL, Akinlade B, Ardeleanu M. Two phase 3 trials of dupilumab versus placebo in atopic dermatitis. N Engl J Med. 2017;376(11):1090-1091.

24. Snast I, Reiter O, Hodak E, Friedland R, Mimouni D, Leshem YA. Are biologics efficacious in atopic dermatitis? A systematic review and meta-analysis. Am J Clin Dermatol. 2018;19(2):145-165.

25. Hwang CY, et al. Prevalence of atopic dermatitis, allergic rhinitis and asthma in Taiwan: a national study 2000 to 2007 . Acta Derm Venereol. 2010;90(6):589-594.

26. Mandelin JM, et al. Long-term efficacy and tolerability of tacrolimus $0.03 \%$ ointment in infants:* a two-year open-label study. Int J Dermatol. 2012;51(1):104-110.

27. Tom WL, Van Syoc M, Chanda S, Zane LT. Pharmacokinetic profile, safety, and tolerability of crisaborole topical ointment, $2 \%$ in adolescents with atopic dermatitis: an open-label phase 2a study. Pediatr Dermatol. 2016;33(2):150-159.

28. Czarnowicki T, Krueger JG, Guttman-Yassky E. Novel concepts of prevention and treatment of atopic dermatitis through barrier and immune manipulations with implications for the atopic march. J Allergy Clin Immunol. 2017;139(6):1723-1734.

29. Myles IA, Reckhow JD, Williams KW, Sastalla I, Frank KM, Datta SK. A method for culturing Gram-negative skin microbiota. BMC Microbiol. 2016;16:60.

30. Arfsten DP, Garrett CM, Jederberg WW, Wilfong ER, McDougal JN. Characterization of the skin penetration of a hydrocarbon-based weapons maintenance oil. J Occup Environ Hyg. 2006;3(9):457-464.

31. Inoshima I, et al. A Staphylococcus aureus pore-forming toxin subverts the activity of ADAM10 to cause lethal infection in 
mice. Nat Med. 2011;17(10):1310-1314.

32. Jensen JM, et al. Impaired sphingomyelinase activity and epidermal differentiation in atopic dermatitis. J Invest Dermatol. 2004;122(6):1423-1431.

33. Kano-Sueoka T, Oda D, Kawamoto JK. Phosphatidylethanolamine deficiency in membrane lipids inhibits keratinocyte intercellular networks formation. In Vitro Cell Dev Biol Anim. 2001;37(10):691-697.

34. Pullmannová P, Staňková K, Pospíšilová M, Skolová B, Zbytovská J, Vávrová K. Effects of sphingomyelin/ceramide ratio on the permeability and microstructure of model stratum corneum lipid membranes. Biochim Biophys Acta. 2014;1838(8):2115-2126.

35. Li S, et al. Altered composition of epidermal lipids correlates with Staphylococcus aureus colonization status in atopic dermatitis. Br J Dermatol. 2017;177(4):e125-e127.

36. Berdyshev E, et al. Lipid abnormalities in atopic skin are driven by type 2 cytokines. JCI Insight. 2018;3(4):e98006.

37. Nakatsuji T, et al. Antimicrobials from human skin commensal bacteria protect against Staphylococcus aureus and are deficient in atopic dermatitis. Sci Transl Med. 2017;9(378):eaah4680.

38. Asher MI, et al. Worldwide time trends in the prevalence of symptoms of asthma, allergic rhinoconjunctivitis, and eczema in childhood: ISAAC Phases One and Three repeat multicountry cross-sectional surveys. Lancet. 2006;368(9537):733-743.

39. Gittler JK, Wang JF, Orlow SJ. Bathing and associated treatments in atopic dermatitis. Am J Clin Dermatol. 2017;18(1):45-57.

40. Olle B. Medicines from microbiota. Nat Biotechnol. 2013;31(4):309-315.

41. Ngugi BM, et al. Effects of bacterial vaginosis-associated bacteria and sexual intercourse on vaginal colonization with the probiotic Lactobacillus crispatus CTV-05. Sex Transm Dis. 2011;38(11):1020-1027.

42. Gerding DN, et al. Administration of spores of nontoxigenic Clostridium difficile strain M3 for prevention of recurrent C. difficile infection: a randomized clinical trial. JAMA. 2015;313(17):1719-1727.

43. Seité S, Zelenkova H, Martin R. Clinical efficacy of emollients in atopic dermatitis patients - relationship with the skin microbiota modification. Clin Cosmet Investig Dermatol. 2017;10:25-33.

44. La Colla L, Mangano A, Mangano A, Albertin A. Effects of nonpathogenic gram-negative bacterium Vitreoscilla filiformis lysate on atopic dermatitis: a prospective, randomized, double-blind, placebo-controlled clinical study. Does this make a real difference? Br J Dermatol. 2009;161(2):477-478.

45. Bin L, Leung DY. Genetic and epigenetic studies of atopic dermatitis. Allergy Asthma Clin Immunol. 2016;12:52.

46. Zhang E, Tanaka T, Tajima M, Tsuboi R, Nishikawa A, Sugita T. Characterization of the skin fungal microbiota in patients with atopic dermatitis and in healthy subjects. Microbiol Immunol. 2011;55(9):625-632.

47. Neuber K, Schwartz I, Itschert G, Dieck AT. Treatment of atopic eczema with oral mycophenolate mofetil. Br J Dermatol. 2000;143(2):385-391.

48. Msika P, De Belilovsky C, Piccardi N, Chebassier N, Baudouin C, Chadoutaud B. New emollient with topical corticosteroidsparing effect in treatment of childhood atopic dermatitis: SCORAD and quality of life improvement. Pediatr Dermatol. 2008;25(6):606-612

49. Pajno GB, et al. Sublingual immunotherapy in mite-sensitized children with atopic dermatitis: a randomized, double-blind, placebo-controlled study. J Allergy Clin Immunol. 2007;120(1):164-170.

50. Janmohamed SR, et al. The proactive wet-wrap method with diluted corticosteroids versus emollients in children with atopic dermatitis: a prospective, randomized, double-blind, placebo-controlled trial. J Am Acad Dermatol. 2014;70(6):1076-1082.

51. Weston S, Halbert A, Richmond P, Prescott SL. Effects of probiotics on atopic dermatitis: a randomised controlled trial. Arch Dis Child. 2005;90(9):892-897. 